\title{
ON THE BACTERIOLOGY OF APICAL ABSCESSES
}

\author{
A Preliminary Report \\ JOSEPH HEAD AND C. ROOS
}

The authors have been engaged in the study of the bacteriology of pyorrhea, and conditions associated with it, for the past five years. Two previous papers-"The bacteriology of pyorrhea alveolaris," by Brown (1) and "The treatment of inflammatory degeneration of the gums and alveolar process," by Head (2)-appeared in 1913. The present paper is a preliminary report of further findings, and is aimed entirely at the apical abscesses where anaerobic conditions prevail. It is hoped that the publication of this preliminary report may serve as a guide to ways and methods comparatively little applied as yet in the exploration of a very important field, and be a gentle challenge and stimulus to those investigators especially who have clinical materiai available in abundance, unexcelled laboratory facilities constantly at their disposal, and to whom superior technique has become a habit. A more complete report is intended shortly, covering the details and results of the other phases of this work, such as serologic tests and classification of the streptococci, on the use of bacterial vaccines, and a discussion and corroboration of the more important literature on the subject.

In our study of over 350 cases of mouth infection associated with pyorrheal pockets, the microorganisms grown aerobically were found to be present in the following percentages of cases: Streptococci, 95 per cent; Gram-negative cocci of the M. catarrhalis group, B. influenzae, and other Gram-negative bacilli of the hemophilic group, about 80 per cent; pneumococci, staphylococci, and bacilli of the diphtheroid group, in a percentage sufficiently large to indicate their probable importance; an occasional scattering of Friedlander's bacillus, M. tetragenus, and a few unidentified organisms. Of course, a study of smears from the mouth secretions and the scum on the teeth might show the presence of any microörganisms found in the air, for mouth 
breathing occurs, uncooked food is eaten, and unboiled water and milk are swallowed.

The organisms mentioned above were obtained from specimens taken from the deeper layers and walls of the infected pockets, and, because they were found either in the depths of the pockets or within the inflamed tissues, were considered of pathological significance. Autogenous bacterial vaccines were prepared from organisms thus obtained, and these vaccines used with excellent results. Gums healed under such treatment far more rapidly than where no such vaccines were used, and the gums remained free from infection to a degree hitherto considered impossible. This was especially noted for cases that, having had purely local treatment for a number of years, showed only slight improvement. The vaccines in these instances caused marked permanent improvement.

A study of $\mathrm{x}$-ray plates indicated that there must be numerous areas of infection absolutely shut off from the air, and it was considered possible, if not probable, that anaerobes might also be the cause of serious pathological lesions. It was therefore decided to carry on some investigations by culturing anaerobically the specimens taken from areas where such conditions were indicated.

The material for the anaerobic cultures was taken as follows: Where $\mathrm{x}$-ray plates showed a necrotic tip, or an absorbed tip of a root having no direct communication with the mouth cavity and this root was to be extracted, an injection of sterile novocain was made. The neck of the tooth was first washed with alcohol until it was considered to be practically free from living bacteria. It was then painted with concentrated tincture of iodine, three times at short intervals, so that it would be practically impossible for any organisms on the neck of the tooth to be alive, care being taken to prevent contamination with saliva. The root was then extracted with sterile forceps and the tip snipped off with a pair of sterile scissors, so that the infected apical portion of the root fell into the culture medium.

The performance of apicoectomy by means of a sterile burr has also occasionally been resorted to. The operation in every case has been performed in a strictly aseptic manner and the end of the burr, with the particles of the material adhering to it, nipped off and dropped directly into the culture tube. 
The culture media, besides the ordinary media used, consisted of sterile tissue medium bouillon overlaid with sterile mineral oil after the method of Noguchi, standard blood-agar plates, dextrose-serum agar and dextrose-serum bouillon; also Heilman's acid bouillon medium for the isolation of the bacilli of the Moro-Tissier group, notably $B$. acidophilus and B. bifidus. The tissue medium tubes, as soon as they reached the laboratory, usually within two or three hours, were incubated at $37^{\circ} \mathrm{C}$. for forty-eight hours, when subcultures were made into the same medium.

Upon repeated examination of a large number of cultures, it was found that, as a rule, the maximum development of all the organisms occurred in the third transplant, especially that of the strictly anaerobic type. Hence, the third transplant was selected for detailed study. Morphology was determined by making slides and staining with differential stains. Plating was conducted, on blood-agar plates, to determine the facultative anaerobic organisms present by growing them under aerobic and anaerobic conditions. Shake-cultures in dextrose-serum agar were poured in heavy plates in order to facilitate the study of the characteristics of growth of the bacterial colonies and isolation of the anaerobic serophilic organisms. Plain, dextrose, or dextrose-serum, bouillon has been used to determine the nutritive requirements of these organisms en masse or in pure cultures, and their acid and gas productions.

The present series comprises a study of 130 specimens from 100 cases. Streptococci were found in 124 of these specimens (alone in 10 , usually a single strain, occasionally two strains; with a few exceptions these were Streptococcus viridans); a minute Gram-negative cocco-bacillus in 90 specimens from as many cases; B. acidophilus and the other members of the Moro-Tissier group in 45 specimens.

In the earlier part of this work, when our technique undoubtedly was lacking in some necessary details, a far greater variety of organisms was usually found to be present. On several occasions of late, where separate cultures have been made from each of the roots of an extracted tooth, the Gram-negative cocco-bacilli and the streptococci have been found in the cultures from roots showing necrosis and absorption, whereas those from the roots that were normal in appearance were usually sterile. 
An abscess or fistula having a more or less direct communication with the mouth cavity, and a variable oxygen tension, invariably shows a larger number and greater variety of organisms than does a closed abscess. Besides the microorganisms mentioned above, leptothrix, B. fusiformis, a Gram-negative bacillus closely resembling the B. rhinitis as described by Tunicliff, Staphylococcus aureus and albus, the diphtheroid bacilli, Gram-negative cocci of the $M$. catarrhalis and M. flavus varieties, Gram-negative hemophilic bacilli of the $B$. influenzae group, and at least two types of Gram-negative cocci, facultative anaerobes, were more or less common in occurrence.

This apparently heretofore undescribed Gram-negative coccobacillus seems to be persistently present in conditions, in 90 cases out of 100 , involving absorption and necrosis of the roots, when the pericementum has been exfoliated to such an extent that the toxins have a free route, due to the almost complete lack of the protective lymphatics, throughout the spongy portions of the alveolar process into the blood supply, where the devastating influences of these toxins are manifest as the cause of various systemic disturbances. In two cases at least this cocco-bacillus was also obtained from the antrum.

Bacterial vaccines containing this organism and streptococci seem to have given far more permanent results, although the dosage had to be greatly reduced. In some instances exceedingly small doses have caused most violent reactions in the patients-reactions that had never before been observed where this microorganism was not used.

This organism, almost constantly present in specimens of this nature, is a Gram-negative, minute, non-motile, organism. No sporeformation has been observed. Smears made without disturbance of the sediment frequently show small clumps of these organisms in a mass. It grows very slowly at first, appearing in large numbers in the second and third transplants made at intervals of forty-eight hours, grown at $37^{\circ} \mathrm{C}$. It does not grow at room-temperature. The organism so far has not been successfully cultivated in pure culture. It is strictly anaerobic and does not survive exposure to aerobic conditions even at incubation temperature on highly nutritive pabulum, for over 24 hours. Heavily seeded blood-agar plates from twenty-four hour 
tissue cultures, when scraped after 24 hours of incubation and these surface scrapings reinoculated into tissue medium, failed to show the development of these organisms. It is proteolytic and produces a certain foul, characteristic odor in tissue media; markedly less so in serum media, growing rather poorly in the latter. The addition of dextrose-serum bouillon does not help to improve its growth. No growth in plain or dextrose bouillon has been noted.

The organism has been found invariably in symbiosis with streptococci, usually the Streptococcus viridans; also quite frequently with one or more of the bacilli of the Moro-Tissier group, mostly the B. acidophilus.

In symbiosis with streptococci, or streptococci and the bacilli of the Moro-Tissier group, this cocco-bacillus has a considerable pathogenic action for the smaller laboratory animals, e.g., mice and guinea pigs. One tenth to one-twentieth of the total amount of growth obtained by centrifugation of a twenty-four hour tissue culture, of the third to the sixth generation, containing about $10 \mathrm{cc}$. of neutral veal-ascites bouillon and a small piece of guinea pig or rabbit kidney, will usually kill a full-grown mouse ( 25 grams) in from sixteen to thirty-six hours after intraperitoneal injection. Cultures made from the heart blood and the peritoneal fluid of the animals treated in this way showed the presence of the Gram-negative cocco-bacilli and streptococci.

The pathogenicity of the streptococci isolated from such specimens, and of the bacilli of the Moro-Tissier group, is very low for laboratory animals, enormous doses, the growths in several culture tubes, being required to kill a mouse by intraperitoneal injections.

The problem of symbiosis will be developed and dealt with more extensively in our completed paper on this subject, as it affords some very interesting and important facts tending towards the solution of the problem of the predisposing causes of certain infectious processes.

\section{LITERATURE CITED}

(1) Brown 1913 New York Medical Journal, 1xi, p. 22, December 20.

(2) HeAD 1913 Journal of the American Medical Association, xcviii, p. 1201, December 20 .

235 South Fifteenth Street, Philadelphia, $P a$.

THE JOURNAL OF DENTAL RESEARCH, VOL. $I$, NO. 